\title{
Can amphiphile architecture directly control vesicle size?
}

\author{
Martin J. Greenall and Carlos M. Marques \\ Institut Charles Sadron, University of Strasbourg, \\ CNRS - UPR 22, 23, rue du Loess, 67034 Strasbourg, France
}

(Dated: January 19, 2013)

\begin{abstract}
Bilayer membranes self-assembled from simple amphiphiles in solution always have a planar ground-state shape. This is a consequence of several internal relaxation mechanisms of the membrane and prevents the straightforward control of vesicle size. Here, we show that this principle can be circumvented and that direct size control by molecular design is a realistic possibility. Using coarse-grained calculations, we design tetrablock copolymers that form membranes with a preferred curvature, and demonstrate how to form low-polydispersity vesicles while suppressing micellization.
\end{abstract}

A fundamental process in soft matter science is the selfassembly of amphiphilic molecules into structures ranging from simple micelles to complex connected aggregates [1-3]. Self-assembled structures not only occur naturally in living cells, but can also be designed for applications such as drug delivery [4]. The question that lies at the heart of this field is how the properties of the individual amphiphilic molecules control the topology of the aggregates they form [5]. One of the major unsolved problems is to design a molecule that can directly fix the curvature of a membrane in solution. In addition to its fundamental interest, this question is of great practical importance, as finding such a molecule would allow the spontaneous formation of vesicles of a well-defined size, yielding precise control of drug delivery systems.

At present, membrane curvature can only be controlled by rather complex procedures. Several of these [6-11] blend two species of amphiphile [12], so that the symmetry of the inner and outer bilayer leaflets is broken [13] and the vesicle has a preferred radius. Such methods have the disadvantage that blends of amphiphiles can form a wide range of micelles, which may coexist with the target vesicle structure [9]. Other methods involve the use of more intricate vesicle formation pathways, such as dewetting from a template [14], cooling and warming through a cylinder-vesicle shape transition [15], electroformation on micropatterned glass slides [16], flow focusing [17], and combined extrusion and dialysis [18].

In this Letter, we investigate an alternative strategy for controlling membrane curvature. We break the membrane symmetry by the use of $\mathrm{ABCA}^{\prime}$ tetrablock copolymers [19-21]. The outer $\mathrm{A}$ and $\mathrm{A}^{\prime}$ blocks of the polymer are formed of the same hydrophilic material, and the B and $\mathrm{C}$ blocks are hydrophobic and have a repulsive interaction with each other. In contrast to the bilayers formed by diblocks (Fig. 1a), these molecules form asymmetric monolayers in solution [19] (Fig. 1b). We use tetrablocks rather than $\mathrm{ABC}$ triblocks since, in this latter case, the $\mathrm{A}$ and $\mathrm{C}$ blocks would have to be hydrophilic and have a strong mutual repulsion for asymmetric monolayers to form. This combination is hard to achieve, both because of the difficulty of finding hydrophilic compounds that repel strongly and the dilution of the hydrophilic layers
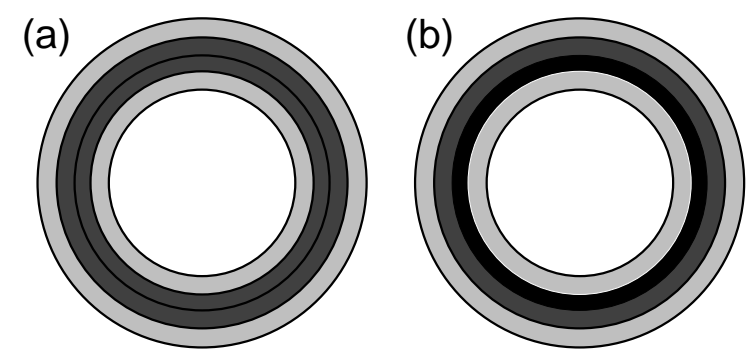

FIG. 1: (a) Equatorial section of a bilayer vesicle of diblock copolymers. Hydrophilic blocks are light gray and hydrophobic blocks are dark gray. (b) Monolayer vesicle of tetrablock copolymers. The hydrophobic blocks are colored black and dark gray, with black block being more hydrophobic.

by solvent, which weakens any interaction between them.

Tetrablock vesicles have indeed been formed in preliminary experimental investigations by Brannan and Bates [19], who also achieved some size control of the aggregates. Such vesicles have also been found in very recent Monte Carlo simulations [21], although this method can only access small vesicles and does not allow size control to be studied. Here, we present a concrete theoretical demonstration of the basic principle that tetrablock copolymers can form bilayers of a preferred curvature, and show how to design these molecules to control the vesicle radius and polydispersity while avoiding micelle formation [20].

We focus on a simple model of $\mathrm{ABCA}^{\prime}$ tetrablocks in A homopolymer 'solvent'. Dilute block copolymerhomopolymer blends provide a good model of aqueous copolymer solutions, and show the same sequence of morphologies as a function of block lengths [22], since the mechanism that drives the shape transitions (the chain crowding in the different layers of the membrane) is the same in both cases. These systems are well-described by SCFT [23]. Furthermore, simple mean-field models of copolymer-homopolymer systems have provided important qualitative insights even into aqueous solutions of small biological molecules, notably the problem of membrane fusion [24, 25].

To begin, we consider copolymers where all four seg- 
ments contain the same number of monomers $N / 4$. For simplicity, the A homopolymer molecules also contain $N / 4$ monomers. The strengths of the interactions between the species are set by Flory $\chi$ parameters. Once two $\chi$ parameters have been chosen, the third must be calculated from a relation involving the polarizabilities of the species [26]. To calculate the density profiles and free energies of the self-assembled structures, we used a simple coarse-grained mean-field theory (self-consistent field theory, or SCFT). The individual polymer molecules interact via a contact potential, and are modeled by random walks, which are averaged over by the SCFT to calculate the density profiles $[27,28]$. SCFT is well-adapted to our current investigation, as its speed allows us to study a much larger range of vesicle sizes than Monte Carlo methods applied to a comparable system [21], and can be nearly as accurate as these more expensive methods for long polymers [29]. The diffusion equations describing the polymers were solved by a finite-difference method and the SCFT equations by an iterative scheme [30], supplemented by extrapolation.

Since we focus on spherical vesicles, we perform many of our calculations assuming spherical symmetry in a spherical box. We must connect the free energy of the subsystem of volume $V$ containing the vesicle to that of the whole system. To do this, we calculate the free-energy density $\tilde{F}$ of a box (with periodic boundary conditions) containing a single spherical vesicle in the canonical ensemble. We then vary the volume of the simulation box at constant overall copolymer volume fraction $\phi[23]$. This corresponds to a larger system (of fixed total volume and fixed copolymer volume fraction) varying the number of aggregates and hence the volume occupied by each. If $\tilde{F}(V)$ has a minimum, this means that the vesicle membranes have an optimum curvature.

This minimum corresponds to the absolute free energy minimum of a solution of spherical vesicles, and a point on $\tilde{F}(V)$ corresponds to a monodisperse solution of vesicles of a given size. We now use these curves to take into account simple fluctuations around the free energy minimum to calculate the polydispersity $\Delta$ of the vesicles. This is related to the free energy $f_{p}$ of an aggregate of $p$ molecules by $1 / \Delta^{2}=\partial^{2} f_{p} / \partial p^{2}$ [31]. To extract this quantity, we write

$\tilde{F}=\left(\phi-v_{\mathrm{m}} / V\right) \ln \left[\left(\phi-v_{\mathrm{m}} / V\right) / e\right]+\left(\phi-v_{\mathrm{m}} / V\right) f_{1}+v f_{p} / V$

where $1 / V$ is the number density of aggregates and $v_{\mathrm{m}}=p v$ is the volume of an aggregate. The first term arises from the entropy of the free copolymers in solution. Now, a single SCFT calculation finds the local free energy minimum $\tilde{F}(V)$ for a vesicle in a box of volume $V$. In the process, it determines the optimum number of molecules in the vesicle for this box size and so corresponds to minimizing $\tilde{F}$ with respect to $p$ at a given $1 / V$. Varying $V$ then yields $\tilde{F}(V)$, from which we can read off

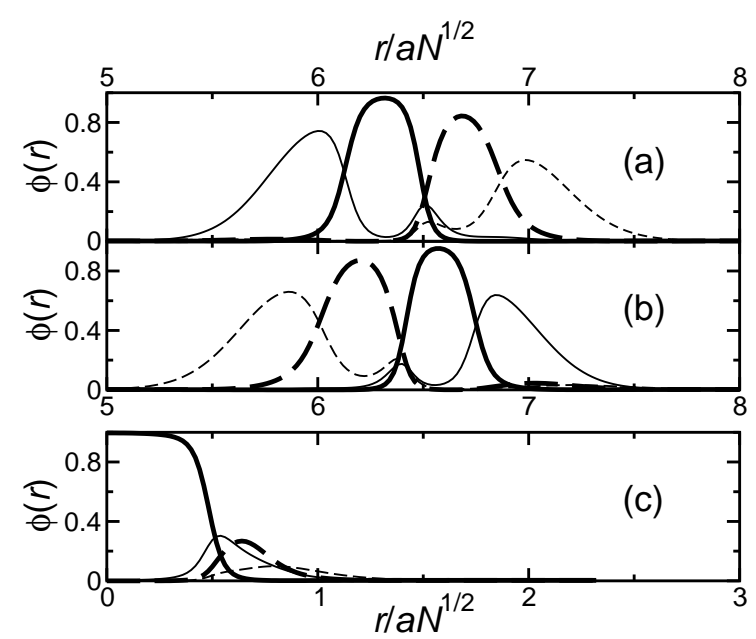

FIG. 2: Sample volume fraction profiles for (a) $\mathrm{ABCA}^{\prime}$ vesicles, (b) vesicles in the $\mathrm{A}^{\prime} \mathrm{CBA}$ orientation, and (c) micelles. A-blocks: thin full lines, B-blocks: thick full lines, C-blocks: thick dashed lines, $\mathrm{A}^{\prime}$ - blocks: thin dashed lines.

$\partial^{2} \tilde{F} / \partial V^{2}$. Remembering that this derivative is evaluated along the line where $\partial \tilde{F} /\left.\partial p\right|_{V}=0$, we find that

$$
\frac{1}{\Delta^{2}}=\frac{\partial^{2} f_{p}}{\partial p^{2}}=\frac{v}{v_{\mathrm{m}}^{2} /\left(V^{3} \partial^{2} \tilde{F} / \partial V^{2}\right)-\left(\phi V-v_{\mathrm{m}}\right)}
$$

allowing us to calculate $\Delta$.

We now demonstrate that the target vesicle structure is a solution to SCFT. In Fig. 2a, we plot cuts through the density profile of an $\mathrm{ABCA}^{\prime}$ vesicle, with $\phi=0.05$. The $\chi$ parameters must be large enough for the amphiphile to aggregate, and so we set $\chi_{\mathrm{AB}}=50 / N$ and $\chi_{\mathrm{AC}}=$ $30 / N$, where $N$ is the total number of monomers in the copolymer. So that the B and $\mathrm{C}$ species demix, we choose the larger of the two possible values [26] for $\chi_{\mathrm{BC}}$, which we set to $157.5 / N$. We measure all lengths in units of $a N^{1 / 2}$, where $a$ is the segment length [28].

The $\mathrm{ABCA}^{\prime}$ structure sketched in Fig. 1 is clearly reproduced in Fig. 2a. The strongly hydrophobic B-blocks lie in the inner half of the membrane, so that the more energetically unfavorable AB interface has a smaller area. Surprisingly, we also find solutions, shown in Fig. 2b, where the B-blocks lie on the outside of the membrane.

In Fig. 2c, we plot the density profile of a micelle, formed in a smaller calculation box. The core is formed from the strongly hydrophobic B blocks. This structure is most likely formed as in ABA triblock micelles [32], with the copolymers forming a hairpin shape.

In Fig. 3, we plot $\tilde{F}$ as a function of the $\mathrm{ABCA}^{\prime}$ vesicle radius $R$ (the radius on the outside of the vesicle at which the copolymer and solvent densities are equal), which we vary by changing $V$ as detailed above. We fix the zero of our free energy scale to correspond to a homogeneouslymixed system of the same composition. The curve shows a minimum as a function of $R$, demonstrating that the 


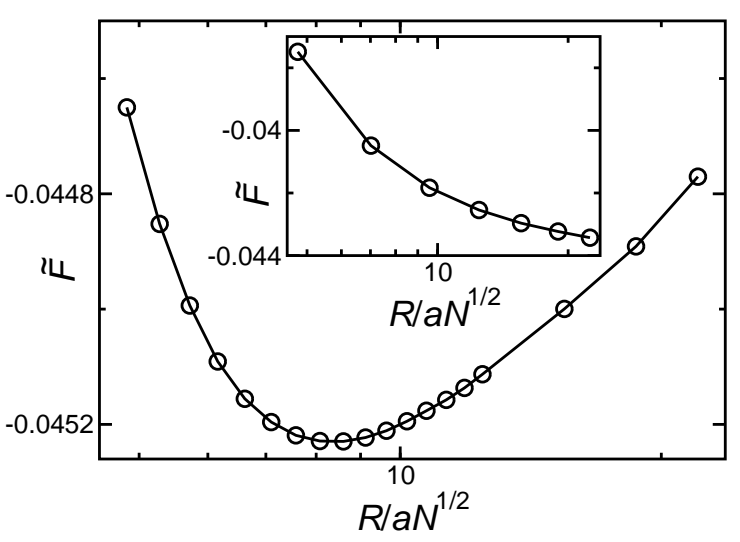

FIG. 3: Free energy density as a function of vesicle radius $R$. The inset shows the corresponding curve for vesicles formed of polymers in the $\mathrm{A}^{\prime} \mathrm{CBA}$ orientation.

vesicles have a preferred size. This is in sharp contrast to the monotonic decrease of the free energy density of the $\mathrm{A}^{\prime} \mathrm{CBA}$ vesicle, plotted in the inset.

To understand this, note that, at smaller radii, the free energy densities of both vesicles decrease with increasing size, as the copolymers are less compressed in the inner leaflet. The $\mathrm{ABCA}^{\prime}$ vesicle always has a lower free energy than the $\mathrm{A}^{\prime} \mathrm{CBA}$ vesicle. As the vesicle radius increases, both membranes become flatter and the relative advantage of the $\mathrm{ABCA}^{\prime}$ vesicle decreases. The two lines then approach each other, with the $\mathrm{ABCA}^{\prime}$ curve now rising and the $\mathrm{A}^{\prime} \mathrm{CBA}$ curve continuing to fall. The monotonic form of the $\mathrm{A}^{\prime} \mathrm{CBA}$ curve is also clear evidence that the $\mathrm{ABCA}^{\prime}$ minimum is not a finite size effect.

To calculate the relative polydispersity of the vesicles, we plot the main free energy curve of Fig. 3 as a function of $V$, and calculate $\partial^{2} \tilde{F} / \partial V^{2}$ at the minimum. We calculate the aggregate volume $v_{\mathrm{m}}$ by integrating over the vesicle density profile and subtracting the local volume fraction at the edge of the system, where it has reached a stable bulk value. Next, we estimate the volume $v$ of a single copolymer molecule. By recalling that all volumes are measured in units of $a^{3} N^{3 / 2}$, and defining the segment volume such that $v=a^{3} N$, we can show that $\Delta / p$ is given by the product of a term specified uniquely by our SCFT calculations and $1 / N^{1 / 4}$. This shows that the polydispersity is rather insensitive to the choice of $N$ within the physical range [20] of $N \sim 100-1000$. Even using the smallest value, $N=100$, we find clear size selection, with $\Delta / p \approx 0.09$. Since the vesicle is relatively flat, we can assume that its surface area is proportional to $p$ and hence that $R \propto p^{1 / 2}$. This yields a relative polydispersity of the radius of 0.05 , and shows that strong size selection takes place in our simple model.

We now investigate how the copolymer architecture controls the optimum vesicle radius. In addition, the free energies of the optimum spherical and cylindrical micelle structures are calculated, and the shape transi-

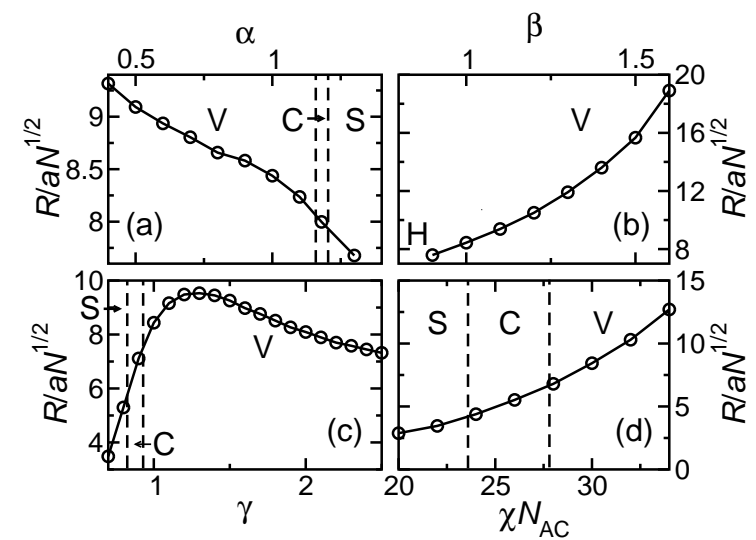

FIG. 4: Vesicle radius as a function of the various block sizes and $\chi_{\mathrm{AC}}$. The radii $R$ are measured in units of $a N^{1 / 2}$, where $N$ is the number of monomers in the original tetrablock copolymers used in the calculations shown in Fig. 2 and Fig. 3. The phase boundaries between the vesicle $(\mathrm{V})$, cylinder (C) and sphere (S) are calculated by interpolating the free energy curves. In the region marked $\mathrm{H}$, the copolymers are homogeneously dispersed in the solvent.

tion boundaries marked on the graph (although only the vesicle radius is shown). First, we multiply the number of hydrophilic monomers in a tetrablock by a factor $\alpha$ (Fig. 4a). The sizes of the $\mathrm{A}$ and $\mathrm{A}^{\prime}$ blocks are changed equally, and the numbers of the hydrophobic $\mathrm{B}$ and $\mathrm{C}$ monomers are left unaltered from our earlier calculations. This procedure follows the experiments of Brannan and Bates [19], and, indeed, we reproduce their result of a decrease in $R$ as $\alpha$ is increased (see Fig. 4a). This is strong evidence that the size selection mechanism at work in their experiments is captured by our model. Furthermore, for $\alpha \approx 1.15$, the free energy of the cylindrical micelle drops below that of the vesicle. This agrees with the experimental observation [19] of the appearance of cylindrical micelles as the A-block length is increased beyond a critical value.

Next, we multiply the number of B-monomers by a factor $\beta$ (Fig. 4b), with the numbers of $\mathrm{A}$ and $\mathrm{C}$ monomers kept constant at the values used in our earlier calculations. For $\beta<0.9$, aggregates of all geometries considered become unstable. As $\beta$ is increased above this value, the vesicle radius grows rapidly, to reduce compression of the B-blocks on the inner membrane surface. The relative polydispersity in the radius falls as $\beta$ increases, reaching 0.03 at $\beta=1.6$. However, this is offset by the fast growth of the vesicle radius itself.

A much more promising route to controlling vesicle size and polydispersity is to vary the less strongly hydrophobic block $\mathrm{C}$ at constant numbers of $\mathrm{A}$ and $\mathrm{B}$ monomers (Fig. 4c). For short C-block lengths $(\gamma<1)$, the repulsion between the two hydrophobic blocks is not sufficiently strong for the well-defined structure shown in Fig. 1 to form. Although vesicles still exist as a solution 
to SCFT, they have higher free energies than micelles and are small and strongly polydisperse. However, as $\gamma$ is increased above unity, the vesicle radius reaches a maximum and then slowly decreases. This can be understood by noting that, once the vesicle structure has been established, the C-blocks lie in the outer half of the vesicle membrane. Increasing the length of the $\mathrm{C}$ blocks further then leads to the formation of smaller, more curved aggregates, to reduce compression of the chains in this outer layer. Furthermore, the relative polydispersity falls as $\gamma$ is increased, remaining close to 0.03 for $\gamma>1.4$. This result shows that, once tetrablock vesicles have been formed in an experiment [19], the formation of small vesicles with a narrow size distribution can be encouraged by lengthening the C-block. We note that the physical mechanism behind this phenomenon is not specific to our current model and can be expected to generalize to systems with other solvents, such as water. An additional benefit of this approach is that, due to the strong repulsion between the long C-block and the other sections of the copolymer, the micellar structures are strongly suppressed, disappearing altogether as solutions to the SCFT at large $\gamma$.

Finally, in Fig. 4d, we show that increasing the repulsion between $\mathrm{A}$ and $\mathrm{C}$ blocks from $\chi_{\mathrm{AC}}=20 / \mathrm{N}$ to $\chi_{\mathrm{AC}}=34 / N$ (which also increases $\chi_{\mathrm{AB}}$ ) produces shape transitions between spherical micelles, cylindrical micelles and vesicles. This demonstrates that the repulsions between the various species must be above a certain threshold for vesicles to form rather than micelles, where the blocks mix in the corona.

In summary, we have demonstrated that the curvature of membranes in solution can be controlled by the architecture of the constituent amphiphilic molecules. We use coarse-grained calculations to show that copolymers composed of two central hydrophobic blocks and two outer hydrophilic blocks form vesicles with a preferred radius. To our knowledge, this is the only system where the molecular structure of the amphiphiles can be shown directly to fix the curvature of a membrane in solution. Control of curvature has only been achieved before by mixing two types of amphiphile or by using a complex self-assembly method. We have reproduced the dependence of vesicle size on hydrophilic block length observed experimentally [19], and have shown how to encourage the formation of vesicles with a narrow size distribution.

The most promising future direction is to focus on the optimum region of parameter space identified here and to include more molecular detail [33], to tune the polymer parameters to produce nearly monodisperse vesicles.

[1] X. He and F. Schmid, Phys. Rev. Lett. 100, 137802 (2008).
[2] S. Jain and F. S. Bates, Science 300, 460 (2003).

[3] L. F. Zhang and A. Eisenberg, Science 272, 1777 (1996).

[4] E. Haleva and H. Diamant, Phys. Rev. Lett. 101, 078104 (2008)

[5] S.-H. Choi, T. P. Lodge, and F. S. Bates, Phys. Rev. Lett. 104, 047802 (2010).

[6] J. H. Lee, V. Agarwal, A. Bose, G. F. Payne, and S. R. Raghavan, Phys. Rev. Lett. 96, 048102 (2006).

[7] E. W. Kaler, A. K. Murthy, B. E. Rodriguez, and J. A. N. Zasadzinski, Science 245, 1371 (1989).

[8] K. Katagiri and F. Caruso, Adv. Mater. 17, 738 (2005).

[9] F. Li, S. Prévost, R. Schweins, A. T. M. Marcelis, F. A. M. Leermakers, M. A. C. Stuart, and E. J. R. Sudhölter, Soft Matter 5, 4169 (2009).

[10] M.-P. Nieh, T. A. Harroun, V. A. Raghunathan, C. J. Glinka, and J. Katsaras, Phys. Rev. Lett. 91, 158105 (2003).

[11] R. Joannic, L. Auvray, and D. D. Lasic, Phys. Rev. Lett. 78, 3402 (1997)

[12] F. Campelo and A. Hernandez-Machado, Phys. Rev. Lett. 100, 158103 (2008).

[13] S. A. Safran, P. Pincus, and D. Andelman, Science 248, 354 (1990).

[14] J. R. Howse, R. A. L. Jones, G. Battaglia, R. E. Ducker, G. J. Leggett, and A. J. Ryan, Nat. Mater. 8, 507 (2009).

[15] A. Rank, S. Hauschild, S. Förster, and R. Schubert, Langmuir 25, 1337 (2009).

[16] P. Taylor, C. Xu, P. D. I. Fletcher, and V. N. Paunov, Chem. Commun. pp. 1732-1733 (2003).

[17] J. Thiele, D. Steinhauser, T. Pfohl, and S. Förster, Langmuir 26, 6860 (2010).

[18] T. F. Zhu and J. W. Szostak, PLoS ONE 4, e5009 (2009).

[19] A. K. Brannan and F. S. Bates, Macromolecules 37, 8816 (2004).

[20] E. D. Gomez, T. J. Rappl, V. Agarwal, A. Bose, M. Schmutz, C. M. Marques, and N. P. Balsara, Macromolecules 38, 3567 (2005).

[21] J. Cui and W. Jiang, Langmuir 27, 10141 (2011).

[22] D. J. Kinning, K. I. Winey, and E. L. Thomas, Macromolecules 21, 3502 (1988).

[23] M. J. Greenall, D. M. A. Buzza, and T. C. B. McLeish, J. Chem. Phys. 131, 034904 (2009).

[24] K. Katsov, M. Müller, and M. Schick, Biophys. J. 87, 3277 (2004).

[25] K. N. Papanicolaou, M. M. Phillippo, and K. Walsh, Am. J. Physio. Heart Circ. Physiol. 303, H243 (2012).

[26] F. Schmid, in Handbook of Multiphase Polymer Systems, edited by A. Boudenne (John Wiley and Sons, Chichester, 2011), chap. 3.

[27] S. F. Edwards, Proc. Phys. Soc. 85, 613 (1965).

[28] M. W. Matsen, in Soft Matter, edited by G. Gompper and M. Schick (Wiley-VCH, Weinheim, 2006), chap. 2.

[29] A. Cavallo, M. Müller, and K. Binder, Macromolecules 39, 9539 (2006).

[30] J. U. Kim and M. W. Matsen, Phys. Rev. Lett. 102, 078303 (2009).

[31] S. Puvvada and D. Blankschtein, J. Chem. Phys. 92, 3710 (1990).

[32] K. Ulrich, P. Galvosas, J. Kärger, and F. Grinberg, Phys. Rev. Lett. 102, 037801 (2009).

[33] S. Pogodin and V. A. Baulin, Soft Matter 6, 2216 (2010). 